\title{
ON THE GENERAL ADDITIVE DIVISOR PROBLEM
}

\author{
ALEKSANDAR IVIĆ AND JIE WU
}

\begin{abstract}
We obtain a new upper bound for $\sum_{h \leqslant H} \Delta_{k}(N, h)$ for $1 \leqslant H \leqslant N$, $k \in \mathbb{N}, k \geqslant 3$, where $\Delta_{k}(N, h)$ is the (expected) error term in the asymptotic formula for $\sum_{N<n \leqslant 2 N} d_{k}(n) d_{k}(n+h)$, and $d_{k}(n)$ is the divisor function generated by $\zeta(s)^{k}$. When $k=3$ the result improves, for $H \geqslant N^{1 / 2}$, the bound given in the recent work [1] of Baier, Browning, Marasingha and Zhao, who dealt with the case $k=3$.
\end{abstract}

\section{In honor of Professor A.A. Karatsuba's 75th birthday}

\section{INTRODUCTION}

Let $d_{k}(n)$ denote that (generalized) divisor function, which represents the number of ways $n$ can be written as a product of $k(\in \mathbb{N})$ factors. Thus

$$
\sum_{n=1}^{\infty} \frac{d_{k}(n)}{n^{s}}=\zeta(s)^{k} \quad(\Re \text { e } s>1),
$$

where $\zeta(s)$ is the familiar zeta-function of Riemann. In particular $d_{1}(n) \equiv 1$ and $d_{2}(n)=\sum_{\delta \mid n} 1$ is the number of positive divisors of $n$. The function $d_{k}(n)$ is a multiplicative function of $n$, and

$$
d_{k}\left(p^{\nu}\right)=(-1)^{\nu}\left(\begin{array}{c}
-k \\
\nu
\end{array}\right)=\frac{k(k+1) \cdots(k+\nu-1)}{\nu !}
$$

for primes $p$ and $\nu \in \mathbb{N}$. The general divisor problem deals with the estimation of $\Delta_{k}(x)$, the error term in the asymptotic formula (see Chapter 13 of Ivić [3] and Chapter 12 of Titchmarsh [15] for an extensive discussion)

$$
D_{k}(x):=\sum_{n \leqslant x} d_{k}(n)=x p_{k-1}(\log x)+\Delta_{k}(x)
$$

where

$$
p_{k-1}(\log x)=\operatorname{Res}_{s=1}\left(\zeta(s)^{k} \frac{x^{s-1}}{s}\right) .
$$

Since $\zeta(s)$ is regular in $\mathbb{C}$ except at $s=1$ where it has a simple pole with residue 1 , it transpires that $p_{k-1}(y)$ is a polynomial of degree $k-1$, whose coefficients may

Date: June 7, 2018.

2000 Mathematics Subject Classification. 11M06.

Key words and phrases. divisor function, general additive divisor problem, power moments, Riemann zeta-function, Ramanujan sum. 
be explicitly evaluated, and in particular $p_{1}(y)=y+2 \gamma-1$, where $\gamma$ is Euler's constant. The important constants $\alpha_{k}, \beta_{k}$ are defined as

$$
\begin{aligned}
\alpha_{k} & :=\inf \left\{a_{k}: \Delta_{k}(x) \ll x^{a_{k}}\right\}, \\
\beta_{k} & :=\inf \left\{b_{k}: \int_{1}^{X}\left|\Delta_{k}(x)\right|^{2} \mathrm{~d} x \ll X^{1+2 b_{k}}\right\} .
\end{aligned}
$$

It is known that $\alpha_{k} \geqslant \beta_{k} \geqslant(k-1) /(2 k)$ for all $k \in \mathbb{N}$, and the conjecture that $\alpha_{k}=\beta_{k}=(k-1) /(2 k)$ for all $k \in \mathbb{N}$ is equivalent to the Lindelöf hypothesis that $\zeta\left(\frac{1}{2}+\mathrm{i} t\right) \ll_{\varepsilon}(|t|+1)^{\varepsilon}$. Here and later $\varepsilon(>0)$ denotes arbitrarily small constants, not necessarily the same ones at each occurrence, while $\ll_{a, b, \ldots}$ means that the implied constant in the $\ll-$ symbol depends on $a, b, \ldots$.

The general additive divisor problem is another important problem involving the divisor function $d_{k}(n)$. It consists of the estimation of the quantity $\Delta_{k}(x, h)$, given by the formula

$$
\sum_{n \leqslant x} d_{k}(n) d_{k}(n+h)=x P_{2 k-2}(\log x ; h)+\Delta_{k}(x, h) .
$$

In (1.4) it is assumed that $k \geqslant 2$ is a fixed integer, and $P_{2 k-2}(\log x ; h)$ is a suitable polynomial of degree $2 k-2$ in $\log x$, whose coefficients depend on $k$ and $h$, while $\Delta_{k}(x, h)$ is supposed to be the error term. This means that we should have

$$
\Delta_{k}(x, h)=o(x) \text { as } x \rightarrow \infty,
$$

but unfortunately (1.5) is not yet known to hold for any $k \geqslant 3$, even for fixed $h$. However, when we consider the sum

$$
\sum_{h \leqslant H} \Delta_{k}(x, h),
$$

we may reasonably hope that a certain cancelation will occur among the individual summands $\Delta_{k}(x, h)$, since there are no absolute value signs in (1.6). It turns out that it is precisely the estimation of the sum in (1.6) which is relevant for bounding the integral

$$
\int_{0}^{T}\left|\zeta\left(\frac{1}{2}+\mathrm{i} t\right)\right|^{2 k} \mathrm{~d} t
$$

which is of great importance in the theory of the Riemann zeta-function (see the monographs $[3,4,15])$.

For $k=1$ the sum in (1.6) is trivial, while for $k=2$ it was extensively studied by many authors, including Kuznetsov [10], Motohashi [13], Ivić \& Motohashi [8] and Meurman [12]. The natural next step in (1.6) is to deal with the case $k=3$, but the works of A.I. Vinogradov and Takhtadžjan [19, 20] and A.I. Vinogradov $[16,17,18]$ show that the analytic problems connected with the Dirichlet series generated by $d_{3}(n) d_{3}(n+h)$ are overwhelmingly hard. The ensuing problems are connected with the group $S L(3, \mathbb{Z})$, and they are much more difficult than the corresponding problems connected with the group $S L(2, \mathbb{Z})$ which appear in the case $k=2$. The latter involve the spectral theory of the non-Euclidean Laplacian, which was extensively developed in recent times by Kuznetsov (see e.g., [11]), Iwaniec and others (see Motohashi's monograph [14] for applications of spectral theory to the 
theory of $\zeta(s))$. Thus at present in the case $k=2$ we have sharp explicit formulas, while in the case $k>2$ we have none.

A.I. Vinogradov [18] conjectured that $\Delta_{k}(x, h) \ll x^{1-1 / k}$, without stating for which range of $h$ this sharp bound should hold. Very likely this bound is too strong, and (even for fixed $h$ ) it seems probable that a power of a logarithm should be included on the right-hand side. More importantly, one hopes that the bound

$$
\sum_{h \leqslant H} \Delta_{k}(x, h) \ll_{k, \varepsilon} H x^{1-1 / k+\varepsilon} \quad \text { for } \quad 1 \leqslant H \leqslant x^{(k-2) / k+\delta_{k}}
$$

holds uniformly in $H$ for fixed $k \geqslant 3$ and some $\delta_{k}>0$, which was stated in [5]. Note that Vinogradov's conjecture in the form $\Delta_{k}(x, h) \ll_{k, \varepsilon} x^{1-1 / k+\varepsilon}$ trivially implies (1.8), but the important point is that there are no absolute value signs in the sum in (1.8). One can also assume (1.8) to hold in the case $k=2$ for $1 \leqslant H \leqslant \sqrt{x}$, say. Then it would follow that the inequality

$$
\int_{T-G}^{T+G}\left|\zeta\left(\frac{1}{2}+\mathrm{i} t\right)\right|^{4} \mathrm{~d} t \ll_{\varepsilon} G T^{\varepsilon}
$$

holds with $G=T^{5 / 6}$, whereas it is known (see e.g., [7]) that $G=T^{2 / 3}$ is unconditionally permissible. It was conjectured in [5] that for any $k \geqslant 2$ and $h \geqslant 1$ one has

$$
\Delta_{k}(x, h)=\Omega\left(x^{1-1 / k}\right) .
$$

For $k=2$ and fixed $h$ this conjecture was proved by Motohashi [13]. As usual, $f(x)=\Omega(g(x))$ means that $\lim _{x \rightarrow \infty} f(x) / g(x) \neq 0$.

The general additive divisor problems is connected to the power moments of $\left|\zeta\left(\frac{1}{2}+\mathrm{i} t\right)\right|$ (see e.g., [3] and [4] for an extensive account). In 1996 the first author [5] proved that

$$
\int_{0}^{T}\left|\zeta\left(\frac{1}{2}+\mathrm{i} t\right)\right|^{6} \mathrm{~d} t \ll_{\varepsilon} T^{1+\varepsilon}+T^{(\alpha+3 \beta-1) / 2+\varepsilon}
$$

provided that

$$
\sum_{h \leqslant H} \Delta_{3}(x, h) \ll_{\varepsilon} H^{\alpha} x^{\beta+\varepsilon}
$$

holds for $1 \leqslant H \leqslant x^{1 / 3+\delta_{3}}$ for some constant $\delta_{3}>0,0 \leqslant \alpha, \beta \leqslant 1, \alpha+\beta \geqslant 1$. The conjecture (1.8) with $k=3$ means that we can take $\alpha=1, \beta=2 / 3$ in (1.11) so that the sixth moment in the form

$$
\int_{0}^{T}\left|\zeta\left(\frac{1}{2}+\mathrm{i} t\right)\right|^{6} \mathrm{~d} t \ll_{\varepsilon} T^{1+\varepsilon}
$$

follows. Note that the best known exponent of $T$ for the right-hand side of the above integral is $5 / 4$ (see [3, Chapter 8]).

In [6] the research begun in [5] was continued, and a plausible heuristic evaluation of the polynomial $P_{2 k-2}(x ; h)$ in $(1.4)$ was made. Yet another (heuristic) evaluation 
of the sum in (1.5) was made later by Conrey and Gonek [2] in 2001. Moreover, it was shown in [6] that, for a fixed integer $k \geqslant 3$ and any fixed $\varepsilon>0$, we have

$$
\int_{0}^{T}\left|\zeta\left(\frac{1}{2}+\mathrm{i} t\right)\right|^{2 k} \mathrm{~d} t \ll_{k, \varepsilon} T^{1+\varepsilon}\left(1+\sup _{T^{1+\varepsilon}<M \ll T^{k / 2}} \frac{G_{k}(M ; T)}{M}\right),
$$

if, for $T^{1+\varepsilon} \leqslant M \ll T^{k / 2}$ and $M<M^{\prime} \leqslant 2 M$,

$$
G_{k}(M ; T):=\sup _{\substack{M \leqslant x \leqslant M^{\prime} \\ 1 \leqslant t \leqslant M^{1+\varepsilon} / T}}\left|\sum_{h \leqslant t} \mathbb{D}_{k}(x, h)\right| .
$$

This result, which generalizes (1.11), provides a directlink between upper bounds for the $2 k$-thmoment of $\left|\zeta\left(\frac{1}{2}+\mathrm{i} t\right)\right|$ and sums of $\mathbb{D}_{k}(x, h)$ over the shift parameter $h$. The result also gives an insight as to the limitations of the attack on the $2 k$-th moment of $\left|\zeta\left(\frac{1}{2}+\mathrm{i} t\right)\right|$ via the use of estimates for $\Delta_{k}(x, h)$. Of course the problem greatly increases in complexity as $k$ increases, and this is one of the reasons why in [5] only the case $k=3$ was considered. The case $k=2$ was not treated, since for the fourth moment of $\left|\zeta\left(\frac{1}{2}+\mathrm{i} t\right)\right|$ we have an asymptotic formula with precise results for the corresponding error term (see e.g., [7] and [14]). Note that (1.13)-(1.14) again lead to the sixth moment bound (1.12) if the conjecture (1.8) holds with $k=3$.

\section{The GENERAL ADDITIVE DIVISOR PROBLEM}

The main objective of this note is to study the averaged sum (1.6), when $k \geqslant 3$ is a fixed integer. To this end we introduce more notation, defining

$$
D_{k}(N, h):=\sum_{N<n \leqslant 2 N} d_{k}(n) d_{k}(n+h)
$$

and letting henceforth

$$
\Delta_{k}(N ; h):=D_{k}(N, h)-\int_{N}^{2 N} \mathfrak{S}_{k}(x, h) \mathrm{d} x,
$$

so that $\Delta_{k}(N ; h)$ in (2.2) differs slightly from (1.4); in fact it equals $\Delta_{k}(2 N, h)-$ $\Delta_{k}(N, h)$ in the notation of (1.4). Here we follow the notation of [1], based on the approach of Conrey and Gonek [2], who made conjectures on the high moments of $\left|\zeta\left(\frac{1}{2}+\mathrm{i} t\right)\right|$. Let us also define

$$
\mathfrak{S}_{k}(x, h):=\sum_{q=1}^{\infty} \frac{c_{q}(h)}{q^{2}} Q_{k}(x, q)^{2}
$$

where $\mu(n)$ is the Möbius function, $c_{q}(h):=\sum_{d \mid(h, q)} d \mu(q / d)$ is the Ramanujan sum and $Q_{k}(x, q)$ is defined as follows. If $\varphi(n)$ is the Euler totient function, set

$$
\Psi_{d, e}(s, q, k):=\frac{d \mu(d) \mu(e)}{\varphi(d) e} \prod_{p \mid(e q / d)}\left\{\left(1-\frac{1}{p^{s}}\right)^{k} \sum_{\nu=0}^{\infty} \frac{d_{k}\left(p^{\nu+\nu_{p}(e q / d)}\right)}{p^{\nu s}}\right\}
$$


where here and later $\nu_{p}(m)$ is the $p$-adic valuation of $m$. Then we define

$$
\begin{aligned}
Q_{k}(x, q) & :=\frac{1}{2 \pi \mathrm{i}} \int_{|s-1|=1 / 8} \zeta(s)^{k} \sum_{d \mid q} \sum_{e \mid d} \Psi_{d, e}(s, q, k)\left(\frac{e x}{d q}\right)^{s-1} \mathrm{~d} s \\
& =\operatorname{Res}_{s=1}\left\{\zeta(s)^{k} \sum_{d \mid q} \sum_{e \mid d} \Psi_{d, e}(s, q, k)\left(\frac{e x}{d q}\right)^{s-1}\right\},
\end{aligned}
$$

by the residue theorem. Thus $Q_{k}(x, q)$ is a polynomial of degree $2 k-2$ whose coefficients depend on $q$, and may be explicitly evaluated. The work of ConreyGonek (op. cit.) predicts, as stated in $(2.2)$, that $D_{k}(N, h)$ is well approximated by $\int_{N}^{2 N} \mathfrak{S}_{k}(x, h) \mathrm{d} x$, which equals $N$ times a polynomial in $\log N$ of degree $2 k-2$, all of whose coefficients depend on $h$ and $k$. This is in agreement with [5] (when $k=3$ and [6] (in the general case), although the shape of the polynomial in question is somewhat different. Conrey and Gonek even predict that uniformly

$$
\Delta_{k}(N ; h) \ll_{\varepsilon} N^{1 / 2+\varepsilon} \quad \text { for } \quad 1 \leqslant h \leqslant N^{1 / 2} .
$$

This conjecture is probably too strong, and one feels that more likely the bound $\Delta_{k}(N ; h) \ll_{\varepsilon} N^{1-1 / k+\varepsilon}$ is closer to the truth (see $(1.10)$ ).

In a recent work [1], Baier, Browning, Marasingha and Zhao obtain new results involving averages of $\Delta_{3}(N ; h)$ (they employ the terminology "shifted convolutions of $d_{3}(n)$ ", which seems appropriate). They proved that

$$
\sum_{h \leqslant H} \Delta_{3}(N ; h) \ll_{\varepsilon} N^{\varepsilon}\left(H^{2}+H^{1 / 2} N^{13 / 12}\right) \quad(1 \leqslant H \leqslant N),
$$

and if $N^{1 / 3+\varepsilon} \leqslant H \leqslant N^{1-\varepsilon}$, then there exists $\delta(\varepsilon)>0$ such that

$$
\sum_{h \leqslant H}\left|\Delta_{3}(N ; h)\right|^{2} \ll_{\varepsilon} H N^{2-\delta(\varepsilon)} .
$$

These results can be used, in conjunction with the bounds (1.13)-(1.14) when $k=3$, to bound the integral in (1.11), but they will produce only the exponent $11 / 8$ on the right-hand side of (1.11), hence no improvement on the known result for the sixth moment of $\left|\zeta\left(\frac{1}{2}+\mathrm{i} t\right)\right|$.

Remark 1. Note that (2.3), in the range $N^{1 / 6+\varepsilon} \leqslant H \leqslant N^{1-\varepsilon}$, provides an asymptotic formula for the averaged sum $\sum_{h \leqslant H} D_{3}(N, h)$ (see $(2.1)$ ). However, it should be noted that no asymptotic formula for the individual $D_{3}(N, h)$ has been found yet, and in general for $\Delta_{k}(N ; h)$ when $k \geqslant 3$. In fact, it is worth pointing out that when $1 \leqslant H \leqslant N^{1 / 6}$, the bound in (2.3) is worse than the trivial bound $H N^{1+\varepsilon}$. Namely we have

$$
\sum_{h \leqslant H} D_{k}(N, h) \ll_{\varepsilon} \sum_{h \leqslant H} \sum_{N<n \leqslant 2 N}(n+h)^{\varepsilon / 2} \ll_{\varepsilon}(H N)^{1+\varepsilon / 2} \ll_{\varepsilon} H N^{1+\varepsilon} .
$$

On the other hand we have

$$
\sum_{h \leqslant H} \int_{N}^{2 N} \mathfrak{S}_{k}(x, h) \mathrm{d} x \ll_{\varepsilon} H N^{1+\varepsilon},
$$


which is obvious from (3.8). Hence by (2.2) it follows that

$$
\sum_{h \leqslant H} \Delta_{k}(N ; h) \ll_{\varepsilon} H N^{1+\varepsilon} \quad(1 \leqslant H \leqslant N),
$$

and clearly (2.5) for $k=3$ improves (2.3) for $1 \leqslant H \leqslant N^{1 / 6}$. The aim of this note is to give a bound for the sum in (1.6), or equivalently for the average of (2.2), which for $k=3$ improves (2.3) for a certain range of $H$. The result is contained in the following

Theorem 1. For fixed $k \geqslant 3$ we have

$$
\sum_{h \leqslant H} \Delta_{k}(N ; h) \ll_{\varepsilon} N^{\varepsilon}\left(H^{2}+N^{1+\beta_{k}}\right) \quad(1 \leqslant H \leqslant N),
$$

where $\beta_{k}$ is defined by (1.3).

Note that we have $\beta_{3}=1 / 3, \beta_{4}=3 / 8$ (see Chapter 13 of [3]), $\beta_{5} \leqslant 9 / 20$ (see Zhang [21]), $\beta_{6} \leqslant 1 / 2$, etc. For a discussion of the values of $\alpha_{k}$ and $\beta_{k}$, see also the paper by Ouellet and Ivić [9].

Corollary 1. . We have, for $1 \leqslant H \leqslant N$,

$$
\begin{aligned}
& \sum_{h \leqslant H} \Delta_{3}(N ; h) \ll_{\varepsilon} N^{\varepsilon}\left(H^{2}+N^{4 / 3}\right), \\
& \sum_{h \leqslant H} \Delta_{4}(N ; h) \ll_{\varepsilon} N^{\varepsilon}\left(H^{2}+N^{11 / 8}\right), \\
& \sum_{h \leqslant H} \Delta_{5}(N ; h) \ll_{\varepsilon} N^{\varepsilon}\left(H^{2}+N^{29 / 20}\right), \\
& \sum_{h \leqslant H} \Delta_{6}(N ; h) \ll_{\varepsilon} N^{\varepsilon}\left(H^{2}+N^{3 / 2}\right) .
\end{aligned}
$$

Remark 2. Since it is known that $\beta_{k}<1$ for any $k$, this means that the bound in (2.6) improves on the trivial bound $H N^{1+\varepsilon}$ in the range $N^{\beta_{k}+\varepsilon} \leqslant H \leqslant N^{1-\varepsilon}$. Our result thus supports the assertion that $\Delta_{k}(N ; h)$ is really the error term in the asymptotic formula for $D_{k}(N, h)$, as given by (3.1) and (3.2). In the case when $k=3$, we have by (2.7) an improvement of (2.3) when $H \geqslant N^{1 / 2}$.

\section{Proof of Theorem 1}

We begin by noting that obviously

$$
\sum_{h \leqslant H} d_{k}(n+h)=\sum_{m \leqslant n+H} d_{k}(m)-\sum_{m \leqslant n} d_{k}(m)
$$

Therefore by (1.1)-(1.2) and (2.1)-(2.2) we can write

$$
\begin{aligned}
\sum_{h \leqslant H} \Delta_{k}(N, h) & =\sum_{N<n \leqslant 2 N} d_{k}(n) \sum_{h \leqslant H} d_{k}(n+h)-\sum_{h \leqslant H} \int_{N}^{2 N} \mathfrak{S}_{k}(x, h) \mathrm{d} x \\
& =M_{k}(N, H)+R_{k}(N, H)-\sum_{h \leqslant H} \int_{N}^{2 N} \mathfrak{S}_{k}(x, h) \mathrm{d} x,
\end{aligned}
$$


say, where

$$
\begin{aligned}
M_{k}(N, H) & :=\sum_{N<n \leqslant 2 N} d_{k}(n) \operatorname{Res}_{s=1}\left(\zeta(s)^{k} \frac{(n+H)^{s}-n^{s}}{s}\right), \\
R(N, H) & :=\sum_{N<n \leqslant 2 N} d_{k}(n)\left(\Delta_{k}(n+H)-\Delta_{k}(n)\right),
\end{aligned}
$$

where $\Delta_{k}(x)$ is defined by (1.1). It is rather easy to estimate $R_{k}(N, H)$. Namely since $d_{k}(n) \ll_{\varepsilon} n^{\varepsilon}$, we have trivially

$$
R_{k}(N, H) \ll_{\varepsilon} N^{\varepsilon} \sum_{n \leqslant 3 N}\left|\Delta_{k}(n)\right| .
$$

For $n<t<n+1$, we obviously have

$$
\Delta_{k}(n)-\Delta_{k}(t)=t p_{k-1}(\log t)-n p_{k-1}(\log n) \ll(\log n)^{k-1} .
$$

Thus

$$
\begin{aligned}
R_{k}(N, H) & \ll_{\varepsilon} N^{\varepsilon} \sum_{n \leqslant 3 N} \int_{n}^{n+1}\left|\Delta_{k}(n)\right| \mathrm{d} t \\
& \ll_{\varepsilon} N^{\varepsilon} \sum_{n \leqslant 3 N} \int_{n}^{n+1}\left|\Delta_{k}(t)\right| \mathrm{d} t+N^{1+\varepsilon} \\
& \ll_{\varepsilon} N^{\varepsilon} \int_{1}^{4 N}\left|\Delta_{k}(t)\right| \mathrm{d} t+N^{1+\varepsilon} \\
& \ll_{\varepsilon} N^{\varepsilon}\left(N \int_{1}^{4 N}\left|\Delta_{k}(t)\right|^{2} \mathrm{~d} t\right)^{1 / 2}+N^{1+\varepsilon} \\
& \ll_{\varepsilon} N^{1+\beta_{k}+\varepsilon},
\end{aligned}
$$

where we used the Cauchy-Schwarz inequality for integrals and the mean square bound (1.3) in the last step.

To estimate $M_{k}(N, H)$, set

$$
u_{k}(x):=\operatorname{Res}_{s=1}\left(\zeta(s)^{k} \frac{(x+H)^{s}-x^{s}}{s}\right) .
$$

Then we can write

$$
M_{k}(N, H)=\int_{N}^{2 N+0} u_{k}(x) \mathrm{d} D_{k}(x)
$$

But we have, since

$$
D_{k}(x)=\operatorname{Res}_{s=1}\left(\zeta(s)^{k} \frac{x^{s}}{s}\right)+\Delta_{k}(x)
$$

in view of (1.1) and (1.2),

$$
M_{k}(N, H)=\int_{N}^{2 N} u_{k}(x) \operatorname{Res}_{s=1}\left(\zeta(s)^{k} x^{s-1}\right) \mathrm{d} x+\int_{N}^{2 N} u_{k}(x) \mathrm{d} \Delta_{k}(x) .
$$


Further note that

$$
\begin{aligned}
& u_{k}(x)=\left.y p_{k-1}(\log y)\right|_{x} ^{x+H} \ll H(\log x)^{k-1}, \\
& u_{k}^{\prime}(x)=\operatorname{Res}_{s=1} \zeta(s)^{k}\left\{(x+H)^{s-1}-x^{s-1}\right\} \ll_{\varepsilon} x^{\varepsilon} .
\end{aligned}
$$

On integrating by parts and using (1.3) and (3.4) we obtain, similarly to (3.2),

$$
\begin{aligned}
\int_{N}^{2 N} u_{k}(x) \mathrm{d} \Delta_{k}(x) & =\left.u_{k}(x) \Delta_{k}(x)\right|_{N} ^{2 N}-\int_{N}^{2 N} \Delta_{k}(x) u_{k}^{\prime}(x) \mathrm{d} x \\
& \ll_{\varepsilon} H N^{\alpha_{k}+\varepsilon}+N^{1+\beta_{k}+\varepsilon} .
\end{aligned}
$$

As for the other integral in (3.3), note that

$$
\frac{(x+H)^{s}-x^{s}}{s}=\frac{x^{s}}{s}\left\{1+\frac{s H}{x}+\left(\begin{array}{l}
s \\
2
\end{array}\right) \frac{H^{2}}{x^{2}}+\cdots-1\right\} .
$$

This gives

$$
\int_{N}^{2 N} u_{k}(x) \operatorname{Res}_{s=1}\left(\zeta(s)^{k} x^{s-1}\right) \mathrm{d} x=H \int_{N}^{2 N}\left(\operatorname{Res}_{s=1} \zeta(s)^{k} x^{s-1}\right)^{2} \mathrm{~d} x+O_{\varepsilon}\left(H^{2} N^{\varepsilon}\right) .
$$

Therefore from (3.3), (3.5) and (3.6) we obtain

$$
\begin{aligned}
M_{k}(N, H)= & H \int_{N}^{2 N}\left(\operatorname{Res}_{s=1} \zeta(s)^{k} x^{s-1}\right)^{2} \mathrm{~d} x \\
& +O_{\varepsilon}\left(H^{2} N^{\varepsilon}+N H^{\alpha_{k}+\varepsilon}+N^{1+\beta_{k}+\varepsilon}\right) .
\end{aligned}
$$

Next we shall prove that

$$
\sum_{h \leqslant H} \int_{N}^{2 N} \mathfrak{S}_{k}(x, h) \mathrm{d} x=H \int_{N}^{2 N}\left(\operatorname{Res}_{s=1} \zeta(s)^{k} x^{s-1}\right)^{2} \mathrm{~d} x+O_{\varepsilon}\left(N^{1+\varepsilon}\right) .
$$

The case of $k=3$ has been treated in [1]. Here we repeat the same argument with some simplification in the general case, obtaining (3.8).

First write

$$
x^{s-1}=\sum_{n=0}^{\infty} \frac{(\log x)^{n}}{n !}(s-1)^{n} .
$$

Since $\Psi_{d, e}(s, q)$ and $(s-1)^{n} \zeta(s)^{k}$ with $n \geqslant k$ are holomorphic for $\Re e s>0$, Cauchy's theorem allows us to deduce that

$$
Q_{k}(x, q)=\frac{1}{2 \pi \mathrm{i}} \sum_{n=0}^{k-1} \int_{|s-1|=1 / 8} \zeta(s)^{k} \sum_{d \mid q} \sum_{e \mid d} \Psi_{d, e}(s, q) \frac{(\log (d x / e q))^{n}}{n !}(s-1)^{n} \mathrm{~d} s .
$$


Clearly for $\Re e s>\frac{1}{2}$, we have

$$
\begin{aligned}
\Psi_{d, e}(s, q) & \ll \frac{d}{\varphi(d) e} \prod_{p \mid(e q / d)}\left\{\left(1+\frac{1}{p^{1 / 2}}\right)^{k} \sum_{\nu=0}^{\infty} \frac{d_{k}\left(p^{\nu+\nu_{p}(e q / d)}\right.}{p^{\nu s}}\right\} \\
& \left.\ll_{\varepsilon} \frac{d}{\varphi(d) e} \prod_{p \mid(e q / d)}\left\{\left(1+\frac{1}{p^{1 / 2}}\right)^{k} p^{\nu_{p}(e q / d) \varepsilon / 4} \sum_{\nu \geqslant 0} \frac{p^{\nu \varepsilon / 4}}{p^{\nu / 2}}\right)\right\} \\
& \ll_{\varepsilon} q^{\varepsilon / 2} .
\end{aligned}
$$

Thus

$$
Q_{k}(x, q) \ll_{\varepsilon, k} q^{\varepsilon}(\log x)^{k-1},
$$

where the implied constant depends only on $\varepsilon$ and $k$.

In view of (3.9) and the bound $\left|c_{q}(h)\right| \leqslant(h, q)$, we have

$$
\begin{aligned}
\sum_{h \leqslant H} \sum_{q>H} \frac{c_{q}(h)}{q^{2}} Q_{k}(x, q)^{2} & \ll(\log x)^{k-1} \sum_{h \leqslant H} \sum_{q>H} \frac{(h, q)}{q^{2-\varepsilon}} \\
& \ll_{\varepsilon, k} H^{\varepsilon}(\log x)^{k-1} .
\end{aligned}
$$

On the other hand, it is well known that $\sum_{h \leqslant q} c_{q}(h)=0$ if $q>1$. From this it is easy to deduce that

$$
\sum_{h \leqslant H} c_{q}(h)= \begin{cases}H+O(1) & \text { if } q=1 \\ O_{\varepsilon}\left(q^{1+\varepsilon}\right) & \text { if } q>1\end{cases}
$$

With the help of this relation and (3.9), we can write

$$
\begin{aligned}
& \sum_{h \leqslant H} \sum_{q \leqslant H} \frac{c_{q}(h)}{q^{2}} Q_{k}(x, q)^{2} \\
& =\{H+O(1)\} Q_{k}(x, 1)^{2}+O\left((\log x)^{k-1} \sum_{1<q \leqslant H} \frac{1}{q^{1-\varepsilon}}\right) \\
& =H\left(\operatorname{Res}_{s=1} \zeta(s)^{k} x^{s-1}\right)^{2}+O\left((\log x)^{k-1} H^{\varepsilon}\right),
\end{aligned}
$$

where we have used the fact that

$$
Q_{k}(x, 1)=\operatorname{Res}_{s=1}\left(\zeta(s)^{k} x^{s-1}\right) \ll_{k}(\log x)^{k-1} .
$$

By combining (3.10) and (3.11), we obtain (3.8).

From (3.1), (3.2), (3.7) and (3.8) we obtain

$$
\sum_{h \leqslant H} \Delta_{k}(N, H) \ll_{\varepsilon} N^{\varepsilon}\left(H^{2}+H N^{\alpha_{k}}+N^{1+\beta_{k}}\right) \quad(1 \leqslant H \leqslant N) .
$$

But we always have

$$
\alpha_{k} \leqslant \frac{1}{2}+\frac{1}{2} \beta_{k}
$$


To see this note that, for $1 \leqslant H \leqslant x$, the defining relation $(1.1)$ and $d_{k}(n) \ll_{\varepsilon} n^{\varepsilon}$ give

$$
\begin{aligned}
\Delta_{k}(x)-\frac{1}{H} \int_{x}^{x+H} \Delta_{k}(y) \mathrm{d} y & =\frac{1}{H} \int_{x}^{x+H}\left(\Delta_{k}(x)-\Delta_{k}(y)\right) \mathrm{d} y \\
& \ll_{\varepsilon} \frac{1}{H} \int_{x}^{x+H}\left\{\left|D_{k}(x)-D_{k}(y)\right|+O\left(x^{\varepsilon}\right)\right\} \mathrm{d} y \\
& \ll_{\varepsilon} H x^{\varepsilon} .
\end{aligned}
$$

This gives, by the Cauchy-Schwarz inequality for integrals and (1.3),

$$
\begin{aligned}
\Delta_{k}(x) & \ll_{\varepsilon} \frac{1}{H} \int_{x}^{x+H}\left|\Delta_{k}(y)\right| \mathrm{d} y+H x^{\varepsilon} \\
& \ll_{\varepsilon} x^{1+\beta_{k}+\varepsilon} H^{-1}+H x^{\varepsilon} \\
& \ll_{\varepsilon} x^{\left(1+\beta_{k}\right) / 2+\varepsilon}
\end{aligned}
$$

with $H=x^{\left(1+\beta_{k}\right) / 2}$. Hence

$$
\Delta_{k}(x) \ll_{\varepsilon} x^{\left(1+\beta_{k}\right) / 2+\varepsilon}
$$

and (3.13) follows. Now in (3.12) we have $H N^{\alpha_{k}} \leqslant H^{2}$ for $H \geqslant N^{\alpha_{k}}$. If $H \leqslant N^{\alpha_{k}}$, then $H N^{\alpha_{k}} \leqslant N^{2 \alpha_{k}} \leqslant N^{1+\beta_{k}}$ by (3.13). Thus the term $H N^{\alpha_{k}}$ in (3.12) can be discarded, and (2.6) follows. This completes the proof of the Theorem.

\section{REFERENCES}

[1] S. Baier, T.D. Browning, G. Marasingha and L. Zhao, Averages of shifted convolutions of $d_{3}(n)$, to appear in Proc. Edinburgh Math. Soc., see preprint at arXiv:1101.5464.

[2] J.B. Conrey and S.M. Gonek, High moments of the Riemann zeta-function, Duke Math. J. 107 (2001), 577-604.

[3] A. Ivić, The Riemann zeta-function, John Wiley \& Sons, New York 1985 (2nd edition. Dover, Mineola, New York, 2003).

[4] A. Ivić, Mean values of the Riemann zeta-function, LN's 82, Tata Inst. of Fundamental Research, Bombay, 1991 (distr. by Springer Verlag, Berlin etc.).

[5] A. Ivić, On the ternary additive divisor problem and the sixth moment of the zeta-function, in "Sieve Methods, Exponential Sums, and their Applications in Number Theory" (eds. G.R.H. Greaves, G. Harman, M.N. Huxley), Cambridge University Press (Cambridge, UK), 1996, 205-243.

[6] A. Ivić, The general additive divisor problem and moments of the zeta-function, in "New Trends in Probablitiy and Statistics (vol. 4)", Analytic and Probabilistic Methods in Number Theory (Proc. Second Inter. Conference in Honour of J. Kubilius, Palanga, Lithuania, 23-27 Sep. 1996, eds. A. Laurinčikas etc.), TEV, Vilnius, Lithuania and VSP, Utrecht-Tokyo, 1997, pp. 69-89.

[7] A. Ivić and Y. Motohashi, The fourth moment of the Riemann zeta-function, J. Number Theory 51 (1995), 16-45.

[8] A. Ivić and Y. Motohashi, On some estimates involving the binary additive divisor problem, Quarterly Journal Math. (Oxford) (2) 46 (1995), 471-483.

[9] A. Ivić and M. Ouellet, Some new estimates in the Dirichlet divisor problem, Acta Arith. 52 (1989), 241-253.

[10] N.V. Kuznetsov, Convolution of the Fourier coefficients of the Eisenstein-Maass series (in Russian), Zap. Naučn. Sem. LOMI AN SSSR 129 (1983), 43-84. 
[11] N.V. Kuznetsov, Petersson hypothesis for forms of weight zero and Linnik's conjecture. Sums of Kloosterman sums, Math. USSR Sbornik 39 (1981), 299-342.

[12] T. Meurman, On the binary additive divisor problem, in "Number theory" Proceedings of the Turku symposium on number theory in memory of Kustaa Inkeri, Jutila, Matti (ed.) et al., Turku, 1999, Walter de Gruyter, Berlin, 2001, 223-246.

[13] Y. Motohashi, The binary additive divisor problem, Ann. Scient. École Norm. Sup, 4e Sér. 27 (1994), 529-572.

[14] Y. Motohashi, Spectral theory of the Riemann zeta-function, Cambridge University Press, Cambridge, 1997.

[15] E.C. Titchmarsh, The theory of the Riemann zeta-function (2nd edition), Oxford University Press, Oxford, 1986.

[16] A.I. Vinogradov, Poincaré series on $S L(3, \mathbb{R})$ (in Russian), Zap. Naučn. Sem. LOMI AN SSSR 160 (1987), 37-40.

[17] A.I. Vinogradov, Analytic continuation of $\zeta_{3}(s, k)$ to the critical strip. Arithmetic part (in Russian), Zap. Naučn. Sem. LOMI AN SSSR 162 (1987), 43-76.

[18] A.I. Vinogradov, The $S L_{n}$-technique and the density hypothesis (in Russian), Zap. Naučn. Sem. LOMI AN SSSR 168 (1988), 5-10.

[19] A.I. Vinogradov and L.A. Takhtadžjan, The theory of Eisenstein series for the group $S L(3, \mathbb{R})$ and its application to a binary problem (in Russian), Zap. Naučn. Sem. LOMI AN SSSR 76 (1978), 5-53.

[20] A.I. Vinogradov and L.A. Takhtadžjan, The zeta-function of an additive divisor problem and the spectral decomposition of the automorphic Laplacian (in Russian), Zap. Naučn. Sem. LOMI AN SSSR 134 (1984), 84-117.

[21] W. Zhang, On the divisor problem, Kexue Tongbao (in Chinese) 33 (1988), 1484-1485.

Katedra Matematike, Rudarsko-geološki Fakultet, Universitet u Beogradu, ĐuŠINA 7, 11000 BEOGRAD, SERBIA

E-mail address: ivic@rgf.bg.ac.rs

School of Mathematics, Shandong University, Jinan, Shandong 250100, China, Institut Elie Cartan Nancy, CNRS, Université Henri Poincaré (Nancy 1), inRia, Boulevard des Aiguillettes, B.P. 239, 54506 Vandeuvre-lès-Nancy, France

E-mail address: wujie@iecn.u-nancy.fr 